\title{
The Use of Vaughan Procedure in Bouveret Syndrome
}

\author{
Fernando Ponce Leon*1, Camila Nascimento Silva Barros de Oliveira², Gustavo Melo da Silva Silva ${ }^{1}$, Márcio José \\ Jamel $^{3}$, Viviane Leite Abud ${ }^{4}$, Rodrigo Martinez ${ }^{3}$ \\ ${ }^{1}$ General Surgeon - Clementino Fraga Filho University Hospital at Universidade Federal do Rio de Janeiro, Brazil \\ ${ }^{2}$ General Surgeon Resident at Clementino Fraga Filho University Hospital, Universidade Federal do Rio de Janeiro, Brazil \\ ${ }^{3}$ Adjunt Professor of the Department of General Surgery, Universidade Federal do Rio de Janeiro, Brazil \\ ${ }^{4}$ Medical Student at Universidade Federal do Rio de Janeiro, Brazil
}

Received: 邦: September 23, 2018; Published: 㘹 October 01, 2018

*Corresponding author: Fernando Ponce Leon, Clementino Fraga Filho University Hospital at Universidade Federal do, Brazil

\section{Introduction}

Bouveret Syndrome is a rare complication in which a gallstone impacts in the duodenum and cause a gastric outlet obstruction. The gallstone passes through a fistula between the gallbladder and adhered portion of gastrointestinal tract. The formation of the fistula occurs after multiple episodes of cholecystitis. The impacted gallstone provoke gallbladder inflammation and pressure through the gallbladder wall causing erosion and allowing the stone to arrive to the duodenum. Gallstone ileus is responsible for 1-4\% of all gastrointestinal obstruction and Bouveret Syndrome represents $2-3 \%$ of those cases [1]. The rarity of the syndrome can be explained by the fact that the majority of stones are small and doesn't cause gastrointestinal obstruction. The stones that can cause obstruction are usually larger than $2 \mathrm{~cm}$ and $50-70 \%$ of gallstone impact in the ileum [2]. Bouveret Syndrome usually affects older women, and many have serious concomitant medical illnesses, including coronary disease, pulmonary disease, and diabetes mellitus that increases mortality and morbidity rates. The symptoms are vague and includes epigastric pain, nausea and vomiting. Less commonly, abdominal pain, distention, upper gastrointestinal bleeding, fever, weight loss and anorexia can be present [1]

\section{Case Report}

A 56 years old female patient, previously diagnosed with cholelithiasis, presented 7 days history with right hypochondrium pain, nausea and multiple vomiting episodes. The patient have been treated in another hospital where she underwent a abdominal Computed Tomography scanning. At the time she did not have the images nor the report. Although the patient referred better control of abdominal pain, she have had three episodes of vomiting on the day before. On physical examination the patient wan in bad condition, with mildly distended abdomen and pain on palpation of the right upper quadrant. Murphy sign was positive. Laboratory examination revealed a white blood cell count of 15.7 (x109/L), alkaline phosphatase 460 and (IU/L) and yGT 45 (UI/L). On abdominal ultrasound the gallbladder was mildly distended, had a thick wall and multiple gallstones. No dilatation of the bile ducts was detected. Surgical treatment was decided.

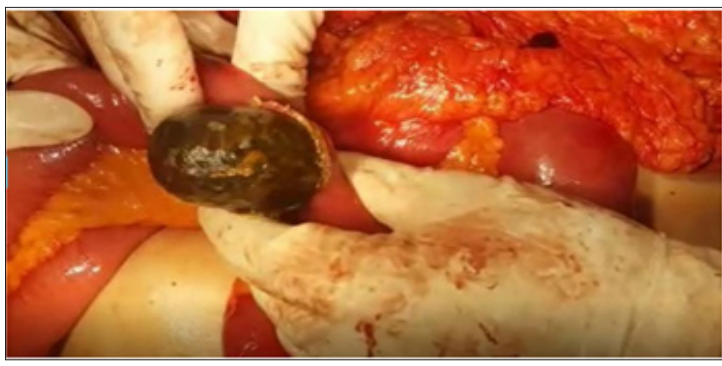

Figure 1: Gallstone extraction.

A laparoscopic surgery was initially performed but the procedure was converted to an open laparotomy due to difficulty in dissection and in visualizing the gallbladder because of the intense inflammatory process and presence of adherences. A duodenal lesion was inadvertently made during the dissection of the gallbladder, allowing the identification of a scleroatrophic gallbladder and a duodenal fistula. The small bowel was explored and a large gallstone was palpated in the 4 th part of the duodenum. An enterotomy and successful removal were performed (Figure 1). The size of the extracted calculus was $5 \mathrm{~cm}$ and no other stones were identified in the digestive tract. The fistula caused great deformity in the duodenum and a pyloric exlusion performed. After surgery the patient needed intensive therapy care due to an episode of bronchoaspiration during the induction of the anesthesia. A small fistula was identified in the gastroenteroanastomosis on the 14 postoperative day. A new surgery was realized and a 20FR Foley catheter was positioned in the fistula. Ten days after, a fistulography was performed. The stomach was visualized full of contrast and no leaking to the cavity was identified (Figure 2). The Foley catheter 
was removed and the patient was sent to the infirmary. In the 35 postoperative day, the patient was discharged. In a consult two months after the surgery, the patient remained asymptomatic.

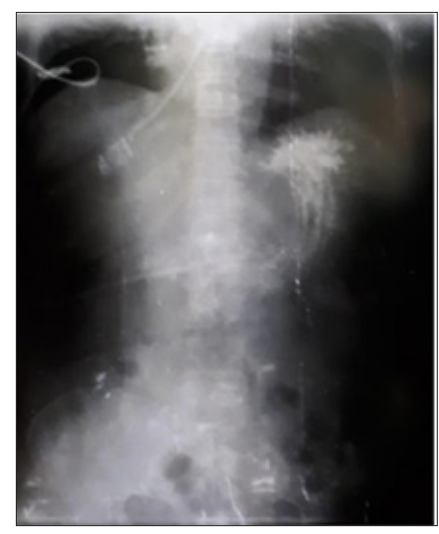

Figure 2: Fistulography by Foley catheter.

\section{Discussion}

The population affected by Bouveret Syndrome usually have advanced age and medical commorbities. Because of that, endoscopic or percutaneous approach like extracorporeal shockwave lithotripsy or laser lithotripsy is being used as first therapeutic approach. In $90 \%$ of the cases, these approaches are unsuccessful and surgery is pursuit for definitive treatment [34]. The surgical options depends on patient condition. High-risk patients with shock or presented with thick adherences should be submitted to enterolithotomy with the extraction of the obstructing gallstone alone [2]. The patients should go through a surveillance period and an election cholecystectomy should be evaluated. Postoperative persistence of symptoms supports the performance of posterior cholecystectomy. Patients with good general status can be submitted to enerolithotomy associated with cholecystectomy and fistula repair. This approach is used in order to prevent new episodes of cholecystitis, cholangitis and development of gallbladder carcinoma [5].

In this approach an increased mortality and morbidity rates can be noticed. The pyloric exclusion reported by Vaughan et al. is performed in high-risk duodenal injury. The procedure consists in primary repair of duodenal wound, closure of the pylorus through an antrum gastrotomy to prevent gastric secretion to arrive in the duodenum and a gastojejunostomy. The management of duodenal injury is controversial. Approximately $70-85 \%$ of duodenal injury can be treated with primary suture [6]. Patients with high-risk lesion have greater risk of developing fistula, abscess and peritonitis and have greater morbi-mortality rates [7]. In these cases, complex repair like duodenal exclusion can be performed. Severe duodenal injury includes bullets wounds, delay to repair exceeding 24 hours, injury of the firs or second parts of the duodenum and duodenal injuries of AAST-OIS grade III and associated injuries to the pancreas [6]. The Vaughan procedure was performed in this case to protect the suture of the duodenum wich was in an area with intense inflammation and extent anatomic destruction. This technique allowed stone extraction and fistula repair in the same operation.

\section{References}

1. Haddad FG, Mansour W, Deeb L (2018) Bouveret's Syndrome: Literature Review 10(3): e2299.

2. (2017) Andrew P Keaveny, Nezam H Afdhal, Steven Bowers, sl Wenliang Chen, Gallstone Ileus.

3. AL Habbal Y, Ng M, Bird D, McQuillan T, AL Khaffaf H (2017) World Uncommon presentation of a common disease - Bouveret's syndrome: A case report and systematic literature review. Journal of Gastrointestinal Surgery 9(1): 25-36.

4. Turner AR, Bhimji SS (2017) Bouveret Syndrome. StatPearls Publishing.

5. Vasileios K Mavroeidis, Dimitrios I Matthioudakis, Nikolaos K Economou, Ioannis D Karanikas (2013) Bouveret Syndrome-The Rarest Variant of Gallstone Ileus: A Case Report and Literature Review.

6. Fraga Gustavo Pereira, Biazotto G, Bortoto JB, Andreollo NA, Mantovani $M(2008)$ The use of pyloric exclusion for treating duodenal trauma: case series. São Paulo Medical Journal 126(6): 337-341.

7. Pierro André Canesso, Mantovani Mario, Reis Norair Salviano dos, Morandin Rosana Celestina, Fraga Gustavo Pereira (2005) Treatment of complex duodenal trauma: comparison between simple suture and suture associated with pyloric exclusion and gastrojejunostomy in dogs. Acta Cir Bras 20(1): 28-38.
ISSN: 2574-1241

DOI: 10.26717/BJSTR.2018.09.001808

Fernando Ponce Leon. Biomed J Sci \& Tech Res

This work is licensed under Creative Commons Attribution 4.0 License

Submission Link: https://biomedres.us/submit-manuscript.php

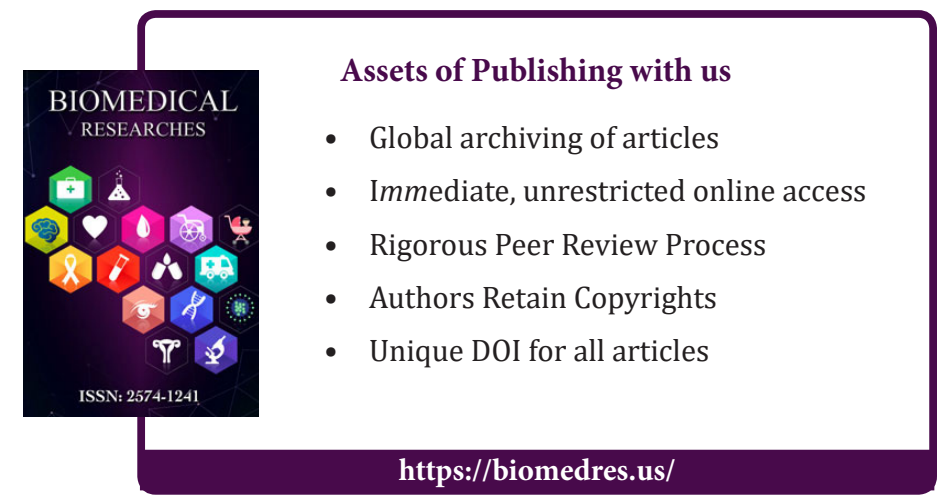

